Józef Myjak · Tomasz Szarek

\title{
On Hausdorff dimension of invariant measures arising from non-contractive iterated function systems
}

Received: May 2, 2001; in final form: July 30, 2001

Published online: May 29, 2002 - (C) Springer-Verlag 2002

Abstract. Under rather general assumptions we give an upper and lower bound estimate of the Hausdorff dimension of the invariant probability measure for an iterated function systems acting on a Polish space.

Mathematics Subject Classification (1991). Primary: 47A35; secondary: 28D15

Key words. Markov operator - Iterated Function System - invariant measure - Hausdorff dimension

\section{Introduction}

Hausdorff dimension, introduced in 1919, is a notion of size useful for distinguishing between sets of Lebesgue measure zero. This notion was widely investigated and widely used, among others in the theory of dynamical systems, where many interesting invariant sets are null in the sense of Lebesgue measure. Unfortunately, the Hausdorff measure and dimension of even relatively simple sets can be very hard to calculate. Several ways (for example, packing dimension, box dimension, informatic dimension, correlation dimension, entropy) of estimating the dimension of irregular sets has been proposed, but undoubtedly the most popular is the Hausdorff dimension.

In 1975 Mandelbrot [19] observed that some scientific phenomena can be described by the sets having fractional dimension. He called such sets fractals and tentatively defined them as the sets whose Hausdorff dimension is strictly greater from their topological dimension. It appears that such sets occur in diverse branches of pure mathematics such as number theory, measure theory, and nonlinear differential equations. On the other hand, these sets are a good model for describing a wide variety of natural phenomena such as Brownian motion, turbulence flow, and the growth of plants. Because of this, the theory of fractals has been intensively studied by many people.

J. Myjak: Dipartamento di Matematica Pura ed Applicata, Universitá di L'Aquila, via Vetoio, 67100 L'Aquila, Italy and WMS AGH al. Mickiewicza 30, 30-059 Kraków, Poland, e-mail: myjak@univaq.it

T. Szarek: Institute of Mathematics Silesian University, Bankowa 14, 40-007 Katowice, Poland and Department of Mathematics Technical University of Rzeszów, W. Pola 6, 35-959 Rzeszów, Poland, e-mail: szarek@ux2 .math.us .udu.pl 
The very explosion of interest in fractal sets started after the observation that some classes of fractals can be constructed by using an iteration process (see Hutchinson [15], Barnsley [1]). More precisely, having $N$ contractive maps, say $S_{1}, \ldots, S_{N}$, we can construct the fractal set $A_{*}$ as the limit of the sequence $\left\{F^{n}(A)\right\}$, where $F(A)=\bigcup_{i=1}^{N} S_{i}(A), A$ a compact set. Moreover, if for every map $S_{i}$ there is given the probability $p_{i}$ determining the frequency with which the map $S_{i}$ can be chosen, we can associate to such a system - known under the name iterated function system with probabilities - a Markov operator $P$ acting on the space of Borel measures (see Formula (1.1)). The fractal set can be obtained as the support of the invariant measure with respect to $P$, provided that $P$ is asymptotically stable. It is interesting that the class of sets which can be defined in this way contains not only fractals but also other sets known as semifractals. Roughly speaking, a semifractal is the support of the invariant measure with respect to an asymptotically stable Markov operator generated by an iterated function system with probabilities (see Lasota and Myjak [17, 18]).

The important question is to find effective criteria for asymptotic stability. Classical results in this area were obtained by Hutchinson [15], Barnsley et al. [2] and Lasota and Yorke [16] in the case of the Menger space (i.e. the space such that every closed bounded subset is compact). Recently such criteria were established in the case of Polish spaces (see Szarek [25, 26]). It seems that natural habitats for fractals and semifractals are Polish spaces. Consequently, the results concerning Hausdorff dimension of invariant measures with respect to Markov operators arising from iterated function systems acting on Polish spaces seem to be of particular interest. For related results see $[6,7,9,11-14,20,22,24]$. These results are formulated for contractive systems and proved using a multifractal formalism.

In this paper we study a more general case when the system is contracting "on the average". More precisely, we give an upper and lower bound estimate of the Hausdorff dimension of the probability measure invariant with respect to the system contractive on the average. Observe that for such system we are unable to set up a one-to-one correspondence between general symbolic dynamical systems and the system under consideration, consequently we cannot use the thermodynamic formalism (see [24]). The approach used here is based on the technique initiated by Hutchinson [15] (see also [2, 11, 12]).

This paper is organized as follows. Notation and basic definitions are contained in Section 1. In Section 2 we establish some auxiliary results which play a crucial role in the proof of the theorems and also seem to be interesting in themselves. In Sections 3 and 4 we give, respectively, upper and lower bound estimates of the Hausdorff dimension of an invariant probability measure for an iterated function system acting on Polish spaces.

\section{Preliminaries}

Throughout this paper $X$ denotes a Polish space, $\rho$ a metric in $X$ and this assumption will not be repeated in the statement of theorems. By $B(x, r)$ we denote the closed ball in $X$ with center at $x$ and radius $r$. For $A \subset X, A \neq \emptyset$, we denote by $\operatorname{diam} A$ 
the diameter of $A$ and by $1_{A}$ the characteristic function of $A$. As usual, $\mathbb{R}$ stands for the set of all reals and $\mathbb{N}$ for the set of all positive integers. Moreover set $\mathbb{R}_{+}=[0,+\infty)$.

By $\mathcal{B}(X)$ we denote the $\sigma$-algebra of Borel subsets of $X$ and by $\mathcal{M}$ the family of all finite Borel measures on $X$. Moreover, denote by $\mathcal{M}_{1}$ the family of all $\mu \in \mathcal{M}$ such that $\mu(X)=1$ and by $\mathcal{M}_{s}=\left\{\mu_{1}-\mu_{2}: \mu_{1}, \mu_{2} \in \mathcal{M}\right\}$ the space of all finite signed measures.

Finally, $B(X)$ stands for the space of all bounded Borel measurable functions $f: X \rightarrow \mathbb{R}$ and $C(X)$ for the subspace of $B(X)$ of all bounded continuous functions. Both spaces are endowed with the norm of uniform convergence $\|\cdot\|_{0}$.

For $f \in B(X)$ and $v \in \mathcal{M}_{s}$ we write

$$
<f, v>=\int_{X} f(x) v(d x) .
$$

We admit that $\mathcal{M}_{S}$ is endowed with the Fortet-Mourier norm (see [10]) given by

$$
\|v\|=\sup \{|<f, v>|: f \in F\},
$$

where $F$ is the set of all functions $f \in C(X)$ such that $\|f\|_{0} \leq 1$ and $|f(x)-f(y)| \leq$ $\rho(x, y)$ for $x, y \in X$.

It is known that the convergence

$$
\lim _{n \rightarrow \infty}\left\|\mu_{n}-\mu\right\|=0 \text { for } \mu_{n}, \mu \in \mathcal{M}_{1}
$$

is equivalent to the weak convergence of the sequence $\left(\mu_{n}\right)_{n \geq 1}$ to $\mu$ (see [4]).

An operator $P: \mathcal{M} \rightarrow \mathcal{M}$ is called a Markov operator if it satisfies the following two conditions:

$$
P\left(\lambda_{1} \mu_{1}+\lambda_{2} \mu_{2}\right)=\lambda_{1} P \mu_{1}+\lambda_{2} P \mu_{2} \text { for } \lambda_{1}, \lambda_{2} \in \mathbb{R}_{+} \text {and } \mu_{1}, \mu_{2} \in \mathcal{M}
$$

and

$$
P \mu(X)=\mu(X) \text { for } \mu \in \mathcal{M} .
$$

A measure $\mu$ is called stationary (or invariant) with respect to the operator $P$ if $P \mu=\mu$. A Markov operator $P$ is called asymptotically stable if there exists an invariant probability measure $\mu_{\star}$ such that

$$
\lim _{n \rightarrow \infty}\left\|P^{n} \mu-\mu_{\star}\right\|=0 \quad \text { for every } \quad \mu \in \mathcal{M}_{1} .
$$

Clearly, if the operator $P$ is asymptotically stable, then the corresponding invariant measure is unique.

Let $A \subset X$ and $s, \delta>0$. Define

$$
\mathscr{H}_{\delta}^{s}(A)=\inf \left\{\sum_{i=1}^{\infty}\left(\operatorname{diam} U_{i}\right)^{s}: A \subset \bigcup_{i=1}^{\infty} U_{i} \text { and } \operatorname{diam} U_{i} \leq \delta\right\} .
$$

Given $s>0$ we define the Hausdorff s-dimensional outer measure by the formula

$$
\mathscr{H}^{s}(A)=\lim _{\delta \rightarrow 0} \mathscr{H}_{\delta}^{s}(A) .
$$


The restriction of $\mathscr{H}^{s}$ to the $\sigma$-algebra of $\mathscr{H}^{s}$-measurable sets is called the Hausdorff s-dimensional measure. Note that all Borel sets are $\mathscr{H}^{s}$-measurable. For $A \subset X$ the value

$$
\operatorname{dim}_{H} A=\inf \left\{s>0: \mathscr{H}^{s}(A)<\infty\right\}
$$

is called the Hausdorff dimension of $A$.

Finally, we recall that the Hausdorff dimension of a Borel probability measure $\mu$ is defined by the formula

$$
\operatorname{dim}_{H} \mu=\inf \left\{\operatorname{dim}_{H} A: A \in \mathcal{B}(X), \mu(A)=1\right\} .
$$

Assume now we are given a sequence of continuous transformations

$$
S_{i}: X \rightarrow X, \quad \text { for } i=1, \ldots, N \text {, }
$$

and a probabilistic vector

$$
p_{i}: X \rightarrow[0,1], \quad \text { for } i=1, \ldots, N,
$$

where $p_{i}$ are continuous functions satisfying

$$
p_{i}(x)>0 \text { and } \sum_{i=1}^{N} p_{i}(x)=1 \text { for } x \in X .
$$

Such a system is denoted by $(S, p)_{N}$ and it is called the Iterated Function System (IFS, for brevity). by

Having an $\operatorname{IFS}(S, p)_{N}$ we define the corresponding Markov operator $P: \mathcal{M} \rightarrow \mathcal{M}$

$$
P \mu(A)=\sum_{i=1}^{N} \int_{S_{i}^{-1}(A)} p_{i}(x) \mu(d x) \quad \text { for } A \in \mathscr{B}(X) .
$$

We say that an IFS $(S, p)_{N}$ is asymptotically stable if the corresponding Markov operator $P$ is asymptotically stable. A measure $\mu_{\star} \in \mathcal{M}$ is called invariant for the IFS $(S, p)_{N}$ if it is invariant with respect to the corresponding Markov operator $P$.

We assume that $S_{i}, i=1, \ldots, N$, are Lipschitzian transformations (not necessarily contractions) with the corresponding Lipschitz constants $\lambda_{i}$ and that there exists a constant $\gamma_{0} \in(0,1)$ such that

$$
\prod_{i=1}^{N}\left(\rho\left(S_{i}(x), S_{i}(y)\right)\right)^{p_{i}(x)} \leq \gamma_{0} \rho(x, y) \quad \text { for every } x, y \in X .
$$

Condition (1.2) is commonly called the average contraction or Lyapunov contraction and the constant $\gamma_{0}$ is strictly connected with Lyapunov exponents (see $\left.[8,23]\right)$ Moreover, we assume that

$$
\kappa=\min _{1 \leq i \leq N} \inf _{x \in X} p_{i}(x)>0
$$


and

$$
\sum_{i=1}^{N}\left|p_{i}(x)-p_{i}(y)\right| \leq \omega(\rho(x, y)),
$$

where $\omega: \mathbb{R}_{+} \rightarrow \mathbb{R}_{+}$is a nondecreasing and concave function satisfying the Dini condition, i.e.

$$
\int_{0}^{\sigma} \frac{\omega(t)}{t} d t<\infty
$$

for some $\sigma>0$.

\section{Auxiliary results}

Proposition 2.1. If an IFS $(S, p)_{N}$ satisfies Conditions (1.2)-(1.4), then it is asymptotically stable.

The proof for the case when $X$ is a locally and $\sigma$-compact metric space was given in $[2,16]$. The case when $X$ is a Polish space it can be found in [26].

Let

$$
\Omega=\{1, \ldots, N\}^{\infty}=\left\{\left(i_{1}, i_{2}, \ldots\right): i_{k} \in\{1, \ldots, N\} \text { for every } k \in \mathbb{N}\right\}
$$

and

$$
\Omega_{*}=\bigcup_{n=1}^{\infty} \Omega_{n}, \text { where } \Omega_{n}=\{1, \ldots, N\}^{n} .
$$

Observe that $\Omega_{*}(\Omega$, respectively) is the space of all finite (infinite, respectively) sequences of elements $i_{k} \in\{1, \ldots, N\}$. For $\mathbf{i}=\left(i_{1}, \ldots, i_{n}\right) \in \Omega_{*}$ let $|\mathbf{i}|=n$ denote the length of $\mathbf{i}$. If $\mathbf{i} \in \Omega$ we assume that $|\mathbf{i}|=\infty$. For $\mathbf{i} \in \Omega \cup \Omega_{*}$ and $m \in \mathbb{N}$, $m \leq|\mathbf{i}|$, we set

$$
\mathbf{i} \mid m=\left(i_{1}, \ldots, i_{m}\right) .
$$

A subset $\Lambda \subset \Omega$ is called a cylinder if there exists $\mathbf{i} \in \Omega_{*}, \mathbf{i}=\left(i_{1}, \ldots, i_{n}\right)$, such that

$$
\Lambda=\Lambda(\mathbf{i})=\{\mathbf{j} \in \Omega: \mathbf{j} \mid n=\mathbf{i}\} .
$$

By $\mathcal{A}$ we denote the $\sigma$-algebra in $\Omega$, which is generated by such cylinders.

Given an IFS $(S, p)_{N}$, for $x \in X$ we denote by $\mathbb{P}_{x}$ the probability measure on $\mathcal{A}$ defined on the cylinder $\Lambda(\mathbf{i}), \mathbf{i}=\left(i_{1}, \ldots, i_{n}\right) \in \Omega_{*}$, by the formula

$$
\mathbb{P}_{x}(\Lambda(\mathbf{i}))=p_{i_{1}}(x) p_{i_{2}}\left(S_{i_{1}}(x)\right) \cdot \ldots \cdot p_{i_{n}}\left(S_{i_{n-1}} \circ \ldots \circ S_{i_{1}}(x)\right) .
$$

For convenience, in the following we will write $\mathbb{P}_{x}(\mathbf{i})$ in the place of $\mathbb{P}_{x}(\Lambda(\mathbf{i}))$ and $\mathbb{P}_{x}(A)$ in the place of $\mathbb{P}_{x}(\Lambda(A))$, where $A \subset \Omega_{n}$ and $\Lambda(A)=\cup_{\mathbf{i} \in A} \Lambda(\mathbf{i})$. Moreover, for $\mathbf{i} \in \Omega_{n}$ we write

$$
S_{\mathbf{i}}=S_{i_{n}} \circ \ldots \circ S_{i_{1}} .
$$

Let $\mu_{*}$ be the unique invariant probability measure for $(S, p)_{N}$. For $\alpha \geq 0$ we define

$$
X_{\alpha}=\left\{x \in X: \liminf _{r \rightarrow 0} \frac{\ln \mu_{*}(B(x, r))}{\ln r} \leq \alpha\right\} .
$$


Lemma 2.2. If an IFS $(S, p)_{N}$ satisfies Conditions (1.2)-(1.4), then

$$
S_{i}\left(X_{\alpha}\right) \subset X_{\alpha},
$$

for every $\alpha \geq 0$ and $i \in\{1, \ldots, N\}$.

Proof. Fix $\alpha \geq 0$ and $i \in\{1, \ldots, N\}$. Let $x \in X_{\alpha}$ be arbitrary. Using the fact that $\mu_{*}$ is stationary, Formula (1.1), Condition (1.3), and the inclusion $B\left(x, r / \lambda_{i}\right) \subset$ $S_{i}^{-1}\left(B\left(S_{i}(x), r\right)\right)$ we obtain

$$
\mu_{*}\left(B\left(S_{i}(x), r\right)\right) \geq \kappa \mu_{*}\left(S_{i}^{-1}\left(B\left(S_{i}(x), r\right)\right)\right) \geq \kappa \mu_{*}\left(B\left(x, r / \lambda_{i}\right)\right),
$$

for every $r>0$. Consequently

$$
\liminf _{r \rightarrow 0} \frac{\ln \mu_{*}\left(B\left(S_{i}(x), r\right)\right)}{\ln r} \leq \liminf _{r \rightarrow 0} \frac{\ln \left[\kappa \mu_{*}\left(B\left(x, r / \lambda_{i}\right)\right)\right]}{\ln r} .
$$

From this and the inequality

$$
\liminf _{r \rightarrow 0} \frac{\ln \mu_{*}\left(B\left(x, r / \lambda_{i}\right)\right)}{\ln \left(r / \lambda_{i}\right)} \leq \alpha,
$$

we have

$$
\liminf _{r \rightarrow 0} \frac{\ln \mu_{*}\left(B\left(S_{i}(x), r\right)\right)}{\ln r} \leq \alpha,
$$

which completes the proof.

Proposition 2.3. Assume that an IFS $(S, p)_{N}$ satisfies Conditions (1.2)-(1.4) and $\mu_{*}$ is the corresponding invariant probability measure. Let $\alpha \geq 0$. If $\mu_{*}\left(X_{\alpha}\right)>0$, then $\mu_{*}\left(X_{\alpha}\right)=1$.

Proof. Let an IFS $(S, p)_{N}$ satisfying Conditions (1.2)-(1.4) be given and let $P$ and $\mu_{*}$ be the corresponding Markov operator and invariant measure, respectively. Consider the measure $\bar{\mu}: \mathcal{B}(X) \rightarrow \mathbb{R}$ given by

$$
\bar{\mu}(A)=\frac{\mu_{*}\left(A \cap X_{\alpha}\right)}{\mu_{*}\left(X_{\alpha}\right)}, \quad \text { for } A \in \mathscr{B}(X) .
$$

We have

$$
\begin{aligned}
P \bar{\mu}(A) & =\sum_{i=1}^{N} \int_{S_{i}^{-1}(A)} p_{i}(x) \bar{\mu}(d x) \\
& =\frac{1}{\mu_{*}\left(X_{\alpha}\right)} \sum_{i=1}^{N} \int_{S_{i}^{-1}(A) \cap X_{\alpha}} p_{i}(x) \mu_{*}(d x) .
\end{aligned}
$$

From Lemma 2.2 it follows that $X_{\alpha} \subset S_{i}^{-1}\left(X_{\alpha}\right)$. Using this inclusion, Relation (1.1), and the fact that $\mu_{*}$ is invariant with respect to $P$, we obtain

$$
\begin{aligned}
P \bar{\mu}(A) & \leq \frac{1}{\mu_{*}\left(X_{\alpha}\right)} \sum_{i=1}^{N} \int_{S_{i}^{-1}\left(A \cap X_{\alpha}\right)} p_{i}(x) \mu_{*}(d x) \\
& =\frac{1}{\mu_{*}\left(X_{\alpha}\right)} \cdot P \mu_{*}\left(A \cap X_{\alpha}\right)=\frac{\mu_{*}\left(A \cap X_{\alpha}\right)}{\mu_{*}\left(X_{\alpha}\right)}=\bar{\mu}(A)
\end{aligned}
$$


for every $A \in \mathscr{B}(X)$. Since $P \bar{\mu} \leq \bar{\mu}$ and $P \bar{\mu}(X)=\bar{\mu}(X)=1, P \bar{\mu}=\bar{\mu}$. From the uniqueness of the invariant probability measure it follows immediately that $\bar{\mu}=\mu_{*}$. Consequently $\mu_{*}\left(X_{\alpha}\right)=1$.

Proposition 2.4. Assume that an IFS $(S, p)_{N}$ satisfies Conditions (1.2)-(1.4) and $\mu_{*}$ is the corresponding invariant measure. Let $\alpha \geq 0$. If $\mu_{*}\left(X_{\alpha}\right)>0$, then $\operatorname{dim}_{H} \mu_{*} \leq \alpha$.

Proof. Let $\alpha \geq 0$ be such that $\mu_{*}\left(X_{\alpha}\right)>0$. By Proposition 2.3 we have $\mu_{*}\left(X_{\alpha}\right)=1$. According to the regularity of $\mu_{*}$ and the Ulam theorem, for every $n \in \mathbb{N}$ we can choose a compact set $K_{n} \subset X_{\alpha}$ such that $\mu_{*}\left(K_{n}\right) \geq 1-1 / n$. Since $\mu_{*}\left(\bigcup_{n=1}^{\infty} K_{n}\right)=1$ and $\operatorname{dim}_{H}\left(\bigcup_{n=1}^{\infty} K_{n}\right)=\sup \left\{\operatorname{dim}_{H} K_{n}: n \in \mathbb{N}\right\}$, to complete the proof it is enough to show that

$$
\operatorname{dim}_{H} K_{n} \leq \alpha, \quad \text { for every } n \in \mathbb{N} .
$$

This can be proved by a standard argument. For details see, for example, [8, Proposition 2.3].

Fix $x_{0} \in X$. Let $\gamma_{0}$ be given by Condition (1.2). Now, for arbitrary $\gamma>\gamma_{0}$ and $n \in \mathbb{N}$ we define the function $\varphi_{n}^{\gamma}: X \rightarrow[0,1]$ by the formula

$$
\varphi_{n}^{\gamma}(x)=\mathbb{P}_{x_{0}}\left(Q_{n}\left(\gamma ; x_{0}, x\right)\right),
$$

where

$$
Q_{n}\left(\gamma ; x_{0}, x\right)=\left\{\mathbf{i} \in \Omega: \rho\left(S_{\mathbf{i} \mid k}(x), S_{\mathbf{i} \mid k}\left(x_{0}\right)\right) \leq \gamma^{k} \rho\left(x, x_{0}\right) \text { for every } k \geq n\right\} .
$$

Lemma 2.5. For every $\gamma>\gamma_{0}$ and $n \in \mathbb{N}$ the function $\varphi_{n}^{\gamma}$ given by (2.1) and (2.2) is Borel measurable. Moreover, for every $x \in X$ the sequence $\left(\varphi_{n}^{\gamma}(x)\right)_{n \geq 1}$ is increasing and

$$
\lim _{n \rightarrow \infty} \varphi_{n}^{\gamma}(x)=1
$$

Proof. Fix $\gamma>\gamma_{0}$ and $n \in \mathbb{N}$. For arbitrary $x \in X$ we have

$$
\begin{aligned}
\varphi_{n}^{\gamma}(x) & =1-\mathbb{P}_{x_{0}}\left(\left\{\mathbf{i} \in \Omega: \rho\left(S_{\mathbf{i} \mid m}(x), S_{\mathbf{i} \mid m}\left(x_{0}\right)\right)>\gamma^{m} \rho\left(x, x_{0}\right) \text { for some } m \geq n\right\}\right) \\
& =1-\sum_{m=n}^{\infty} \sum_{\mathbf{i} \in \Omega_{m}} \mathbb{P}_{x_{0}}(\mathbf{i}) 1_{X_{\mathbf{i}}}(x),
\end{aligned}
$$

where $X_{\mathbf{i}}, \mathbf{i} \in \Omega_{m}$, stands for the set of all $y \in X$ such that $\rho\left(S_{\mathbf{i}}(y), S_{\mathbf{i}}\left(x_{0}\right)\right)>$ $\gamma^{m} \rho\left(y, x_{0}\right)$ and $\rho\left(S_{\mathbf{i} \mid k}(y), S_{\mathbf{i} \mid k}\left(x_{0}\right)\right) \leq \gamma^{k} \rho\left(y, x_{0}\right)$ for $k=1, \ldots, m-1$.

Since the sets $X_{\mathbf{i}}, \mathbf{i} \in \Omega_{*}$, are Borel measurable, the function $\varphi_{n}^{\gamma}$ is Borel measurable. Obviously $\varphi_{n}^{\gamma}(x) \leq \varphi_{n+1}^{\gamma}(x)$ for every $n \in \mathbb{N}$ and $x \in X$. Moreover, from Lemma 2 in [5] it follows that $\lim _{n \rightarrow \infty} \varphi_{n}^{\gamma}(x)=1$ for every $x \in X$. The proof is complete.

From Lemma 2.5 and the theorem of Egorov it follows immediately that: 
Corollary 2.6. Let $\gamma>\gamma_{0}$ and $x_{0} \in X$ be given. Then for every $\varepsilon>0$ there exist a subset $X_{0}$ of $X$ and a number $\tilde{n}$ such that

$$
\mu_{*}\left(X_{0}\right)>1-\varepsilon,
$$

and

$$
\mathbb{P}_{x_{0}}\left(Q_{n}\left(\gamma ; x_{0}, x\right)\right) \geq 1-\varepsilon, \quad \text { for every } x \in X_{0} \text { and } n \geq \tilde{n} .
$$

Now for $\gamma>\gamma_{0}, x_{0}, x \in X$ and $n_{0}, n \in \mathbb{N}, n \geq n_{0}$, we define

$$
Q_{n_{0}}^{n}\left(\gamma ; x_{0}, x\right)=\left\{\mathbf{i} \in \Omega_{n}: \rho\left(S_{\mathbf{i} \mid k}(x), S_{\mathbf{i} \mid k}\left(x_{0}\right)\right) \leq \gamma^{k} \rho\left(x, x_{0}\right) \text {, for } n_{0} \leq k \leq n\right\} .
$$

Lemma 2.7. Assume that a compact subset $K$ of $X$, a point $x_{0} \in K$ and a number $n_{0} \in \mathbb{N}$ are given. Then for every $\gamma>\gamma_{0}$ there exist $\beta>0$ such that for every $x \in K$ and $n \geq n_{0}$ we have

$$
\mathbb{P}_{x}(\mathbf{i}) \geq \beta \mathbb{P}_{x_{0}}(\mathbf{i}), \quad \text { for every } \quad \mathbf{i} \in Q_{n_{0}}^{n}\left(\gamma ; x_{0}, x\right) .
$$

Proof. Fix a compact set $K \subset X$ and set $d=\operatorname{diam} K$. Since the function $\omega$ given by (1.4) satisfies the Dini condition, we have (see $[5,16]$ )

$$
\omega_{0}=\sum_{k=1}^{\infty} \omega\left(\gamma^{k} d\right)<\infty
$$

Let $n \geq n_{0}$ and $x \in K$ be arbitrary. For $\mathbf{i} \in Q_{n_{0}}^{n}\left(\gamma ; x_{0}, x\right)$ we have

$$
\begin{aligned}
\mathbb{P}_{x_{0}}(\mathbf{i})= & p_{i_{1}}\left(x_{0}\right) p_{i_{2}}\left(S_{i_{1}}\left(x_{0}\right)\right) \cdot \ldots \cdot p_{i_{n}}\left(S_{\mathbf{i} \mid n-1}\left(x_{0}\right)\right) \\
= & \frac{p_{i_{1}}\left(x_{0}\right) \cdot \ldots \cdot p_{i_{n_{o}}}\left(S_{\mathbf{i} \mid n_{o}-1}\left(x_{0}\right)\right)}{p_{i_{1}}(x) \cdot \ldots \cdot p_{i_{n_{o}}}\left(S_{\mathbf{i} \mid n_{o}-1}(x)\right)} \cdot p_{i_{1}}(x) \cdot \ldots \cdot p_{i_{n_{o}}}\left(S_{\mathbf{i} \mid n_{o}-1}(x)\right) \\
& \cdot \prod_{k=n_{o}+1}^{n}\left(1+\frac{p_{i_{k}}\left(S_{\mathbf{i} \mid k-1}\left(x_{0}\right)\right)-p_{i_{k}}\left(S_{\mathbf{i} \mid k-1}(x)\right)}{p_{i_{k}}\left(S_{\mathbf{i} \mid k-1}(x)\right)}\right) \cdot p_{i_{k}}\left(S_{\mathbf{i} \mid k-1}(x)\right)
\end{aligned}
$$

Using Conditions (1.3) and (1.4), and the definition of $Q_{n_{0}}^{n}\left(\gamma ; x_{0}, x\right)$ we obtain

$$
\begin{aligned}
\mathbb{P}_{x_{0}}(\mathbf{i}) & \leq \frac{(1-\kappa)^{n_{0}}}{\kappa^{n_{0}}} \cdot \prod_{k=n_{0}+1}^{n}\left(1+\frac{\omega\left(\rho\left(S_{\mathbf{i} \mid k-1}(x), S_{\mathbf{i} \mid k-1}\left(x_{0}\right)\right)\right)}{\kappa}\right) \cdot \mathbb{P}_{x}(\mathbf{i}) \\
& \leq\left(\frac{1-\kappa}{\kappa}\right)^{n_{0}} \prod_{k=n_{0}+1}^{n}\left(1+\frac{\omega\left(\gamma^{k-1} d\right)}{\kappa}\right) \cdot \mathbb{P}_{x}(\mathbf{i}) .
\end{aligned}
$$

Consequently

$$
\mathbb{P}_{x_{0}}(\mathbf{i}) \leq\left(\frac{1-\kappa}{\kappa}\right)^{n_{0}} \prod_{k=n_{0}+1}^{\infty} e^{\omega\left(\gamma^{k-1} d\right) / \kappa} \cdot \mathbb{P}_{x}(\mathbf{i}) \leq\left(\frac{1-\kappa}{\kappa}\right)^{n_{0}} e^{\omega_{0} / \kappa} \cdot \mathbb{P}_{x}(\mathbf{i}) .
$$

Setting $\beta=\kappa^{n_{0}}(1-\kappa)^{-n_{0}} e^{-\omega_{0} / \kappa}$ we complete the proof. 
Using a standard martingale argument one can prove the following version of the Brin-Katok theorem (see [3])

Lemma 2.8. Let $x_{0} \in X$. Assume that an IFS $(S, p)_{N}$ satisfies Conditions (1.2)(1.4). Then

$$
\liminf _{n \rightarrow \infty} \frac{1}{n} \ln \mathbb{P}_{x_{0}}(\mathbf{i} \mid n) \geq \ln \delta_{0} \quad \mathbb{P}_{x_{0}} \text {-a.s. }
$$

where

$$
\delta_{0}=\inf _{x \in X} \prod_{i=1}^{N} p_{i}(x)^{p_{i}(x)}
$$

Proof. Define the function $X_{n}: \Omega \rightarrow \mathbb{R}$ by the formula

$$
X_{n}(\mathbf{i})=-\ln \left(p_{i_{n}}\left(S_{i_{n-1}} \circ \ldots \circ S_{i_{1}}\left(x_{0}\right)\right)\right), \quad \text { for } n \in \mathbb{N} \text {. }
$$

For $\mathbf{i} \in \Omega_{*}, \mathbf{i}=\left(i_{1}, \ldots, i_{n}\right)$, we denote by $\mathcal{A}(\mathbf{i})$ the $\sigma$-algebra generated by the cylinders $\left\{\Lambda(\mathbf{j}): \mathbf{j} \in \Omega_{*}, \mathbf{j}>\mathbf{i}\right\}$. Moreover, let $\mathbb{E}_{x_{0}}$ denote the expectation with respect to the probability measure $\mathbb{P}_{x_{0}}$ on $\Omega$.

Fix $\mathbf{i} \in \Omega, \mathbf{i}=\left(i_{1}, i_{2}, \ldots\right)$ and observe that

$$
\mathbb{E}_{x_{0}}\left(X_{n} \mid \mathcal{A}\left(i_{1}, \ldots, i_{n-1}\right)\right)=\sum_{i=1}^{N} p_{i}\left(S_{i_{n-1}} \circ \ldots \circ S_{i_{1}}\left(x_{0}\right)\right) X_{n}\left(\left(i_{1}, \ldots, i_{n-1}, i\right)\right) .
$$

By (2.4) we have

$$
-\sum_{i=1}^{N} p_{i}\left(S_{i_{n-1}} \circ \ldots \circ S_{i_{1}}\left(x_{0}\right)\right) \ln \left(p_{i}\left(S_{i_{n-1}} \circ \ldots \circ S_{i_{1}}\left(x_{0}\right)\right)\right) \leq-\ln \delta_{0} .
$$

Now let $Y_{n}=X_{n}-\mathbb{E}_{x_{0}}\left(X_{n} \mid \mathcal{A}\left(i_{1}, \ldots, i_{n-1}\right)\right)$. Then

$$
\left|Y_{n}(\mathbf{i})\right| \leq 2 \sup _{\mathbf{i} \in \Omega}\left|X_{n}(\mathbf{i})\right| \quad \mathbb{P}_{x_{0}}-\text { a.s. }
$$

Write

$$
M=2 \sup _{\mathbf{i} \in \Omega}\left|X_{n}(\mathbf{i})\right|<\infty .
$$

Define

$$
Z_{n}=\sum_{k=1}^{n} \frac{Y_{k}}{k}, \quad \text { for } n \in \mathbb{N} .
$$

It is easy to see that $\left(Z_{n}\right)_{n \geq 1}$ is a martingale. Since $Y_{k}$ and $Y_{l}$ for $k \neq l$ are mutually orthogonal, we have

$$
\mathbb{E}_{x}\left(Z_{n}^{2}\right) \leq M^{2} \sum_{k=1}^{\infty} \frac{1}{k^{2}}
$$


Hence $\left(Z_{n}\right)_{n \geq 1}$ is an $\mathbb{L}^{2}$-bounded martingale, and so $\left(Z_{n}\right)_{n \geq 1}$ is convergent a.s. Then by Kronecker's lemma (see [4])

$$
\lim _{n \rightarrow \infty} \frac{1}{n} \sum_{k=1}^{n} Y_{k}=0 \quad \mathbb{P}_{x_{0}} \text {-a.s. }
$$

Thus

$$
\underset{n \rightarrow \infty}{\limsup } \frac{1}{n} \sum_{k=1}^{\infty} X_{k}+\ln \delta_{0} \leq 0 \quad \mathbb{P}_{x_{0}} \text {-a.s. }
$$

whence

$$
\liminf _{n \rightarrow \infty} \frac{1}{n} \ln \mathbb{P}_{x_{0}}(\mathbf{i} \mid n) \geq \ln \delta_{0} \quad \mathbb{P}_{x_{0}} \text { a.s. }
$$

The proof is complete.

Finally observe that $-\ln \delta_{0}$ is some form of entropy for the IFS (see [8]).

\section{An upper estimation}

Theorem 3.1. Assume that an IFS $(S, p)_{N}$ satisfies Conditions (1.2)-(1.4) and let $\mu_{*}$ be the corresponding invariant probability measure. Then

$$
\operatorname{dim}_{H} \mu_{*} \leq \frac{\ln \delta_{0}}{\ln \gamma_{0}}
$$

where $\gamma_{0}$ and $\delta_{0}$ are given by (1.2) and (2.4), respectively.

Proof. Let $\mu_{*}$ be the unique invariant probability measure for $(S, p)_{N}$ and let $K$ be a compact subset of $X$ such that $\mu_{*}(K) \geq 3 / 4$. Set $d=\operatorname{diam} K$. Choose $x_{0} \in K$ and let $\delta \in\left(0, \delta_{0}\right)$ and $\gamma \in\left(\gamma_{0}, 1\right)$ be arbitrary. By virtue of Corollary 2.6 there exist a subset $K_{0}$ of $K$ and a number $n_{0} \in \mathbb{N}$ such that $\mu_{*}\left(K_{0}\right) \geq 1 / 2$ and

$$
\mathbb{P}_{x_{0}}\left(Q_{n}\left(\gamma ; x_{0}, x\right)\right) \geq \frac{3}{4}, \quad \text { for every } x \in K_{0} \text { and } n \geq n_{0} .
$$

By virtue of Lemma 2.8 and the theorem of Egorov we can also simultaneously assume that

$$
\mathbb{P}_{x_{0}}\left(\left\{\mathbf{i} \in \Omega: \mathbb{P}_{x_{0}}(\mathbf{i} \mid n) \geq \delta^{n} \text { for every } n \geq n_{0}\right\}\right) \geq 3 / 4 .
$$

From (3.1), (3.2), and the obvious inclusion $Q_{n_{0}}\left(\gamma ; x_{0}, x\right) \subset \Lambda\left(Q_{n_{0}}^{n}\left(\gamma ; x_{0}, x\right)\right)$, $n \geq n_{0}$, it follows that for every $x \in K_{0}$ and every $n \geq n_{0}$ we have

$$
\mathbb{P}_{x_{0}}\left(\left\{\mathbf{i} \in \Omega: \mathbf{i} \mid n \in Q_{n_{0}}^{n}\left(\gamma ; x_{0}, x\right) \text { and } \mathbb{P}_{x_{0}}(\mathbf{i} \mid n) \geq \delta^{n}\right\}\right) \geq \frac{1}{2},
$$

or equivalently that

$$
\sum_{\mathbf{i} \in Q_{n_{0}}^{n}\left(\gamma ; x_{0}, x\right), P_{x_{0}}(\mathbf{i}) \geq \delta^{n}} \mathbb{P}_{x_{0}}(\mathbf{i}) \geq \frac{1}{2} \quad \text { for } x \in K_{0} \text { and } n \geq n_{0} .
$$


Since $Q_{n_{0}}^{n}\left(\gamma ; x_{0}, x\right) \subset Q_{m}^{n}\left(\gamma ; x_{0}, x\right)$ for $n \geq m \geq n_{0}$, by Lemma 2.7 there exists $\beta>0$ such that

$$
\sum_{\mathbf{i} \in Q_{n_{0}}^{n}\left(\gamma ; x_{0}, x\right),} \mathbb{P}_{x}(\mathbf{i}) \geq \frac{\beta}{2}, \quad \text { for } x \in K_{0} \text { and } n \geq n_{0} .
$$

Now, for $n \in \mathbb{N}$ we define

$$
Y_{n}=\bigcup_{\mathbf{i} \in \Omega_{n}, \mathbb{P}_{x_{0}}(\mathbf{i}) \geq \delta^{n}} B\left(S_{\mathbf{i}}\left(x_{0}\right), \gamma^{n} d\right) .
$$

Let $n \geq n_{0}$. For $x \in K_{0}$ and $\mathbf{i} \in Q_{n_{0}}^{n}\left(\gamma ; x_{0}, x\right)$ we have $\rho\left(S_{\mathbf{i}}(x), S_{\mathbf{i}}\left(x_{0}\right)\right) \leq$ $\gamma^{n} \rho\left(x, x_{0}\right) \leq \gamma^{n} d$, which means that $S_{\mathbf{i}}(x) \in Y_{n}$. Consequently, for arbitrary $n \geq n_{0}$ we have

$$
K_{0} \subset S_{\mathbf{i}}^{-1}\left(Y_{n}\right), \quad \text { for every } \mathbf{i} \in Q_{n_{0}}^{n}\left(\gamma ; x_{0}, x\right) .
$$

Since $\mu_{*}$ is an invariant measure for the IFS $(S, p)_{N}$, using (1.1), (3.3), (3.4), and the inequality $\mu_{*}\left(K_{0}\right) \geq 1 / 2$ we obtain

$$
\begin{aligned}
\mu_{*}\left(Y_{n}\right) & =\sum_{\mathbf{i} \in \Omega_{n}} \int_{X} 1_{Y_{n}}\left(S_{\mathbf{i}}(x)\right) \mathbb{P}_{x}(\mathbf{i}) \mu_{*}(d x) \\
& \geq \sum_{\mathbf{i} \in \Omega_{n}, \mathbb{P}_{x_{0}}(\mathbf{i}) \geq \delta^{n}} \int_{X} 1_{Y_{n}}\left(S_{\mathbf{i}}(x)\right) \mathbb{P}_{x}(\mathbf{i}) \mu_{*}(d x) \\
& \geq \int_{K_{0}} \sum_{\mathbf{i} \in Q_{n_{0}}^{n}\left(\gamma ; x_{0}, x\right), \mathbb{P}_{x_{0}}(\mathbf{i}) \geq \delta^{n}} \mathbb{P}_{x}(\mathbf{i}) \mu_{*}(d x) \geq \frac{\beta}{2} \cdot \frac{1}{2}=\frac{\beta}{4}
\end{aligned}
$$

for every $n \geq n_{0}$.

Set $\eta=\beta / 4$. For $n \in \mathbb{N}$ define

$$
Z_{n}=\left\{x \in Y_{n}: \mu_{*}\left(B\left(x, 2 \gamma^{n} d\right)\right) \leq \eta \delta^{n} / 2\right\} .
$$

If $x \in Z_{n}$, by the definition of $Y_{n}$ there exists $\mathbf{i}_{0} \in \Omega_{n}$ with $\mathbb{P}_{x_{0}}\left(\mathbf{i}_{0}\right) \geq \delta^{n}$, such that $x \in B\left(S_{\mathbf{i}_{0}}\left(x_{0}\right), \gamma^{n} d\right)$. Since $B\left(S_{\mathbf{i}_{0}}\left(x_{0}\right), \gamma^{n} d\right) \subset B\left(x, 2 \gamma^{n} d\right)$, it follows that $\mu_{*}\left(B\left(S_{\mathbf{i}_{0}}\left(x_{0}\right), \gamma^{n} d\right)\right) \leq \eta \delta^{n} / 2$. This means that the set $Z_{n}$ can be covered by the balls $B\left(S_{\mathbf{i}}\left(x_{0}\right), \gamma^{n} d\right)$ with $\mathbf{i} \in \Omega_{n}, \mathbb{P}_{x_{0}}(\mathbf{i}) \geq \delta^{n}$ and $\mu_{*}\left(B\left(S_{\mathbf{i}}\left(x_{0}\right), \gamma^{n} d\right)\right) \leq \eta \delta^{n} / 2$. From this and the equality $\mathbb{P}_{x_{0}}(\Omega)=1$ it follows that

$$
\mu_{*}\left(Z_{n}\right) \leq \operatorname{card}\left\{\mathbf{i} \in \Omega_{n}: \mathbb{P}_{x_{0}}(\mathbf{i}) \geq \delta^{n}\right\} \cdot \frac{\eta \delta^{n}}{2} \leq \delta^{-n} \cdot \frac{\eta \delta^{n}}{2}=\frac{\eta}{2},
$$

for every $n \geq n_{0}$. (Here the symbol card stands for the cardinality.) From (3.5) and (3.6) it follows that $\mu_{*}\left(Y_{n} \backslash Z_{n}\right) \geq \eta / 2$ for every $n \geq n_{0}$. Set $X_{n}=Y_{n} \backslash Z_{n}$ and define $X^{\infty}=\bigcap_{m=n_{0}}^{\infty} \bigcup_{n=m}^{\infty} X_{n}$. Since $\mu_{*}\left(X_{n}\right) \geq \eta / 2$ for $n \geq n_{0}$, from the continuity of measure it follows immediately that $\mu_{*}\left(X^{\infty}\right) \geq \eta / 2$. Moreover, a simple calculation shows that

$$
X^{\infty} \subset\left\{x \in X: \liminf _{r \rightarrow 0} \frac{\ln \mu_{*}(B(x, r))}{\ln r} \leq \frac{\ln \delta}{\ln \gamma}\right\} .
$$


Thus, by Proposition 2.4 we have

$$
\operatorname{dim}_{H} \mu_{*} \leq \frac{\ln \delta}{\ln \gamma}
$$

Letting $\delta \rightarrow \delta_{0}$ and $\gamma \rightarrow \gamma_{0}$, we complete the proof.

\section{A lower estimation}

In this section we assume that $S_{i}: X \rightarrow X, i=1, \ldots, N$ are continuous transformations such that

$$
\rho\left(S_{i}(x), S_{i}(y)\right) \geq \ell_{i} \rho(x, y), \quad \text { for } \quad x, y \in X,
$$

where $l_{i} \in(0,1), i=1, \ldots, N$. Without any loss of generalisation we can assume that

$$
l_{1} \leq l_{i} \leq l_{N}, \quad \text { for } \quad i=1, \ldots, N .
$$

Moreover, we assume that there exists a compact set $K$ such that

$$
K=\bigcup_{i=1}^{N} S_{i}(K)
$$

and

$$
\bigcap_{i=1}^{N} S_{i}(K)=\emptyset
$$

Now, for $x \in X$, define

$$
I_{x}=\left\{i: i \in\{1, \ldots, N\} \text { and } x \in S_{i}(K)\right\} .
$$

Theorem 4.1. Assume that an IFS (S, $p)_{N}$ satisfies Conditions (4.1)-(4.4). Moreover, assume that it admits an invariant probability measure $\mu_{*}$ such that supp $\mu_{*}$ $\subset K$. If

$$
\sum_{i \in I_{x}} p_{i}\left(S_{i}^{-1}(x)\right)<1, \quad \text { for every } \quad x \in K
$$

then

$$
\operatorname{dim}_{H} \mu_{*} \geq s_{*},
$$

where

$$
s_{*}=\sup \left\{s>0: \sum_{i \in I_{x}} p_{i}\left(S_{i}^{-1}(x)\right) l_{i}^{-s} \leq 1 \text { for } x \in K\right\},
$$

and $I_{x}$ is defined by (4.5). 
Proof. Consider the function $\sigma: X \rightarrow \mathbb{R}_{+}$given by

$$
\sigma(x)=\min _{i \notin I_{x}} \rho\left(x, S_{i}(K)\right) .
$$

Simple calculation shows that $\sigma$ is a continuous function. Moreover, from (4.4) it follows that $\sigma(x)>0$ for $x \in K$. Thus

$$
\sigma_{0}=\min _{x \in K} \sigma(x)>0
$$

Now, let $s_{*}$ be given by (4.8). Fix an arbitrary $s \in\left(0, s_{*}\right)$. Clearly

$$
\sum_{i \in I_{x}} p_{i}\left(S_{i}^{-1}(x)\right) l_{i}^{-s}<1 \quad \text { for every } \quad x \in K
$$

Owing to the continuity of $p_{i}, S_{i}$ for $i=1, \ldots, N$ and compactness of $K$, there is $\hat{r} \in\left(0, \sigma_{0}\right)$ such that

$$
\sum_{i \in I_{x}} p_{i}\left(z_{i}\right) l_{i}^{-s}<1
$$

for every $x \in K$ and every $z_{i} \in B\left(S_{i}^{-1}(x), \hat{r}\right)$ with $i \in I_{x}$.

Set $r_{0}=l_{1} \hat{r}$. We claim that

$$
\mu_{*}(B(x, r)) \leq\left(\frac{r}{r_{0}}\right)^{s}
$$

for every $x \in K$ and $r>0$.

Obviously (4.10) holds for every $x \in X$ and $r \geq r_{0}$. Define

$$
r_{*}=\inf \left\{r>0: \mu_{*}(B(x, t)) \leq\left(\frac{t}{r_{0}}\right)^{s} \text { for } t \geq r \text { and } x \in K\right\} .
$$

We will prove that $r_{*}=0$. Suppose, for a contradiction, that $r_{*}>0$. Let $r \in$ $\left[l_{N} r_{*}, r_{0}\right)$. Using the fact that $\mu_{*}$ is an invariant measure, the inclusion supp $\mu_{*} \subset K$, Formula (1.1), and the inequality $r<r_{0}$, we have

$$
\mu_{*}(B(x, r))=\sum_{i=1}^{N} \int_{S_{i}^{-1}(B(x, r))} p_{i}(z) \mu_{*}(d z)=\sum_{i=1}^{N} \int_{S_{i}^{-1}(B(x, r)) \cap K} p_{i}(z) \mu_{*}(d z) .
$$

There exists $y \in K$ such that $S^{-1}(B(x, r)) \cap K=\emptyset$ for $i \notin I_{y}$. Hence

$$
\begin{aligned}
\mu_{*}(B(x, r)) & \leq \sum_{i \in I_{y}} \int_{S_{i}^{-1}(B(x, r)) \cap K} p_{i}(z) \mu_{*}(d z) \\
& =\sum_{i \in I_{y}} p_{i}\left(z_{i}\right) \mu_{*}\left(S_{i}^{-1}(B(x, r))\right),
\end{aligned}
$$


for some $z_{i} \in B\left(S_{i}^{-1}(x), r / l_{i}\right), i \in I_{x}$. Since $S_{i}^{-1}(B(x, r) b i g) \subset B\left(S_{i}^{-1}(x), r / l_{i}\right)$ and $r / l_{i}>r_{*}$ we have

$$
\begin{aligned}
\mu_{*}(B(x, r)) & \leq \sum_{i \in I_{x}} p_{i}\left(z_{i}\right) \mu_{*}\left(B\left(S_{i}^{-1}(x), r / l_{i}\right)\right) \leq \sum_{i \in I_{x}} p_{i}\left(z_{i}\right)\left(\frac{r}{l_{i} r_{0}}\right)^{s} \\
& \leq\left(\frac{r}{r_{0}}\right)^{s} \sum_{i \in I_{x}} p_{i}\left(z_{i}\right) l_{i}^{s} .
\end{aligned}
$$

From the last inequality and (4.9) it follows that

$$
\mu_{*}(B(x, r)) \leq\left(\frac{r}{r_{0}}\right)^{s}, \text { for } r \geq l_{N} r_{*} \text { and } x \in K,
$$

which contradicts the definition of $r_{*}$. Thus $r_{*}=0$ and (4.10) hold for every $x \in K$ and $r>0$. Consequently, by the mass distribution principle (see [24, p. 43]) we have

$$
\operatorname{dim}_{H} \mu_{*} \geq s .
$$

Since $s \in\left(0, s_{*}\right)$ was arbitrary, Inequality (4.7) follows. The proof is completed.

Acknowledgements. T. Szarek wishes to thank the University of L'Aquila, where the paper was written, for their invitation and hospitality. He was also supported by the State Committee for Scientific Research (Poland) Grant No. 2 P03A 01016.

\section{References}

1. Barnsley, M. F.: Fractals Everywhere. Boston: Academic Press 1993

2. Barnsley, M.F., Demko, S.G., Elton, J.H., Geronimo, J.S.: Invariant measures arising from iterated function systems with place dependent probabilities. Ann. Inst. Henri Poincaré 24, 367-394 (1988)

3. Brin, M., Katok, A.: On local entropy in geometric dynamics. Lecture Notes in Mathematics 1007, pp. 30-38. Berlin, New York: Springer 1983

4. Dudley, R.M.: Probabilities and Matrics. Aarhus: Aarhus Universitet 1976

5. Elton, J.H.: An ergodic theorem for iterated maps. J. Ergodic Theor. Dynam. Syst. 7, 481-488 (1987)

6. Falconer, K.J.: Dimensions and measures of quasi self-similar sets. Proc. Amer. Math. Soc. 106, 543-554 (1989)

7. Falconer, K.J.: The Geometry of Fractal Sets. Cambridge 1985

8. Falconer, K.J.: Techniques in Fractal Geometry. Wiley 1997

9. Fan, A.H., Lau, K.S.: Iterated function system and Ruelle operator. J. Math. Anal. Appl. 231, 319-344 (1999)

10. Fortet, R., Mourier, B.: Convergence de la répartition empirique vers la répartition théorétique. Ann. Sci. École Norm. Sup. 70, 267-285 (1953)

11. Geronimo, J.S., Hardin, D.P.: An exact formula for the measure dimensions associated with a class of piecewise linear maps. Constr. Approx. 5, 89-98 (1989)

12. Halsey, T.C., Jensen, M.H., Kadanoff, L.P., Procaccia, I., Shraiman, B.J.: Fractal measures and their singularities: The characterization of strange sets. Phys. Rev. A 33, 1141-1151 (1986)

13. Hata, M.: On the structure of self-similar sets. Japan J. Appl. Math. 2, 381-414 (1985)

14. Hata, M.: Mathematics of Fractals. Translation of Mathematical Monogr. 167, Am. Math. Soc. 1997 
15. Hutchinson, J.: Fractals and self-similarity. Indiana Univ. Math. J. 30, 713-747 (1981)

16. Lasota, A., Yorke, J.A.: Lower bound technique for Markov operators and iterated function systems. Random Comput. Dynam. 2, 41-77 (1994)

17. Lasota, A., Myjak, J.: Semifractals on Polish spaces. Bull. Pol. Ac.: Math. 46, 179-196 (1998)

18. Lasota, A., Myjak, J.: Fractals, semifractals and Markov operators. Int. J. Bif. Chaos 9 46, 307-325 (1999)

19. Mandelbrot, B.B.: The Fractals Geometry of Nature. San Francisco: Freeman 1982

20. McLaughlin, J.: A note on Hausdorff measures of quasi self-similar sets. Proc. Amer. Math. Soc. 100, 183-186 (1981)

21. Myjak, J., Szarek, T.: Capacity of an invariant measure. Anna. Polon. Math. 78, 141-157 (2002)

22. Olsen, L.: A multifractal formalism. Adv. Math. 116, 82-195 (1995)

23. Pellicot, M.: Lectures on Pesin Theory and Ergodic Theory on Manifolds. Cambridge: Cambridge University Press 1992

24. Pesin, Y.: Dimension Theory in Dynamical Systems. Chicago: The University of Chicago Press 1997

25. Szarek, T.: Markov operators acting on Polish spaces. Ann. Polon. Math. 67, 247-257 (1997)

26. Szarek, T.: Invariant measures for Markov operators with applications to function systems. Studia Math. To appear

27. Szarek, T.: The dimension of self-similar measures. Bull. Pol. Acad. Sci., Math. 48, 293-302 (2002) 